\title{
Middleware framework for integration of heterogeneous hardware and software
}

\begin{abstract}
There is a significant amount of research on the integration of heterogeneous hardware and software concerning wireless sensor networks (WSN). Due to the limitations in terms of energy, memory capacity and computational power of the wireless sensors, various applications are designed to optimize the use of wireless sensors according to the needs of various business and industries such as environmental monitoring, asset tracking, health monitoring, military surveillance and industrial automation and control. These applications sometimes interacts with one another hence lays the need to integrate data between the applications. The hardware itself is configured based on the requirements of the aforementioned applications considering the limitations of the sensors as various communication protocols and operating systems are used in different types of sensors. As a result of the heterogeneous nature of the hardware and applications concerning wireless sensor networks, a middleware is needed to reduce the effort and cost of integrating the heterogeneous hardware and software. This paper proposes an architectural design of a middleware that tackles the issues of data formatting for hardware and software integration and heterogeneous data integration concerning wireless sensor networks.
\end{abstract}

Keyword: Middleware; Integration; Wireless sensor network; Heterogeneous data 